\title{
ANÁLISIS SOBRE LA IMPORTANCIA DE LA SEGURIDAD Y SALUD EN EL TRABAJO EN EL SECTOR DE LA CONSTRUCCIÓN EN COLOMBIA
}

\author{
Analysis on the importance of safety and health at work \\ in the construction sector in Colombia
}

\author{
CARLOS ALEJANDRO NUÑEZ ARTEAGA ${ }^{1}$ \\ Recibido:5 de diciembre de 2020. Aceptado:13 de enero de 2021 \\ DOI: http://dx.doi.org/10.21017/rimci.2021.v8.n15.a91
}

\begin{abstract}
Resumen
Nos encontramos frente a uno de los sectores económicos que más accidentes de trabajo reporta de manera anual a las administradoras de riesgos laborales en nuestro país, el sector de la construcción. Por medio de un análisis estadístico apoyado en cifras entregadas por la Federación de aseguradores de Colombia - Fasecolda, argumentamos la necesidad que tiene nuestro país de hacer especial énfasis en la interventoría en seguridad y salud en el trabajo en el sector de la construcción, sector que a pesar de presentar índices altos de mortalidad, especialmente en la mano de obra no calificada, no logra visualizar un compromiso claro y responsable por parte de los empleadores, en este caso las empresas constructoras y el ente regulador, que para el caso de nuestro país viene siendo el ministerio de trabajo al momento de realizar la implementación y posterior seguimiento a las actividades encaminadas a salvaguardar la integridad física de obreros y operarios, aun cuando existen estrategias de gestión y una normatividad que apoya dichos procesos desde el punto de vista jurídico.
\end{abstract}

Palabras clave. Estadísticas; riesgos; accidentalidad; interventoría; norma.

\begin{abstract}
We are facing one of the economic sectors that annually reports the most occupational accidents to occupational risk managers in our country, the construction sector. Through a statistical analysis supported by figures provided by the Federation of Insurers of Colombia - Fasecolda, we argue the need for our country to place special emphasis on the supervision of occupational health and safety in the construction sector, a sector that Despite presenting high mortality rates, especially in unskilled labor, it fails to visualize a clear and responsible commitment on the part of employers, in this case the construction companies and the regulatory body, which in the case of our country lt has been the Ministry of Labor when carrying out the implementation and subsequent monitoring of the activities aimed at safeguarding the physical integrity of workers and operators, even though there are management strategies and regulations that support said processes from a legal point of view.
\end{abstract}

Key words. Statistics; risk; accidents; auditing; standard.

\section{INTRODUCCIÓN}

L A INDUSTRIA de la construcción en Colombia, es uno de los principales sectores económicos pero de la misma manera es uno de los sectores que mayores índices de accidentalidad aporta a las estadísticas no gratas del país. De acuerdo a los datos estadísticos entregados año tras año por la Federación de aseguradores de Colombia [1] entre el año 2017 y lo corrido del 2020, el sector de la construcción reporto a las administradoras de riesgos laborales 284.860 accidentes, de los cuales 322 fueron mortales.

1 Facultad de ingeniería y ciencias agroindustriales, Instituto tolimense de formación técnica profesional "ITFIP", Espinal, Tolima - Colombia. Correo electrónico: canunez01@itfip.edu.co. ORCID: https:/orcid.org/0000-0002-2596-7264 
Pese a ser uno de los principales sectores económicos, ya que en la última década ha sido la rama económica que ha aportado más al PIB nacional, siendo una de las mayores industrias, también es uno de los sectores que mayores índices de accidentalidad reporta en el país [2], ubicándose desde el año 2017 en el cuarto sector económico que mayor accidentes de trabajo reporta a las ARL, superado por el sector de la Agricultura, ganadería, caza y silvicultura, la industria manufacturera y los servicios sociales y de salud.

Por este motivo el foco de la problemática se centra principalmente en los trabajadores, sus empleadores y las estrategias que se están llevando a cabo en el campo de la construcción para salvaguardar la salud e integridad física de quienes por pasión, vocación o simplemente necesidad, exponen su vida a diario para llevar el sustento a sus hogares, cumplir objetivos, metas y cronogramas de trabajo a como dé lugar.

Teniendo en cuenta la responsabilidad civil que adquieren las empresas constructoras al momento de contratar mano de obra especializada y no especializada, recurrimos a la normatividad legal vigente en nuestro país para temas como la Seguridad y Salud en el Trabajo, Seguridad Industrial o el concepto más conocido como Salud Ocupacional el cual ya se encuentra derogado por el ministerio de salud [3]. El sector de la construcción cuenta con un conjunto de riesgos laborales propios de la actividad, los cuales son pertinentes al trabajo realizado en altura, labores de excavación, el izado de materiales y el carácter temporal de sus centros de trabajo, entre otros; lo anterior conlleva a que la ejecución del sistema de gestión de la seguridad y salud en el trabajador de dicho sector, sea aplicado de manera diferente [4]. Por lo tanto, es correcto pensar que las actividades que se llevan a cabo en las obras de construcción son consideradas de alto riesgo y por consiguiente pueden desencadenar accidentes de trabajo.

\section{Objetivos}

\section{A. Objetivo General}

Analizar la importancia de la seguridad y salud en el trabajo en el sector de la construcción en Colombia.

\section{B. Objetivos específicos}

- Estudiar las referencias disponible en materia de seguridad y salud en el trabajo en el sector de la construcción, desde el punto de vista de legal, investigativo y programas de prevención que permitan dar el contexto del desarrollo del tema en Colombia.

- Establecer y desarrollar un trabajo investigativo en el que se entienda de una mejor manera los Sistemas de Gestión de Seguridad y Salud en el Trabajo y los accidentes laborales presentados dentro de un periodo de tiempo establecido, para el sector de la construcción.

- Identificar las diferentes actividades en materia de seguridad y salud en el trabajo que llevan a cabo las empresas constructoras colombianas.

\section{MARCO TEÓRICO}

Nos encontramos finalizando el año 2020, año en el que nos vimos golpeados por la pandemia ocasionada por el virus SARS COV 2 que derivó en la enfermedad ya más que conocida COVID-19. Enfermedad que traigo a colación para el presente artículo debido a que por las restricciones emanadas por el gobierno nacional a las diferentes actividades productivas del país con llevo a una disminución considerable en los índices de accidentalidad no solo para el sector de la construcción si no para los demás sectores económicos del país, un alivio en cifras de accidentalidad y mortalidad laboral que pagamos a nivel mundial en materia sanitaria por el desconocimiento y la no aplicación de una cultura preventiva y de autocuidado.

Cifras que para el sector de la construcción en el año 2019 como nos muestra Fasecolda en su informe estadístico anual [1], mostró una disminución con respecto a la accidentalidad presentada en el año 2018 pero que aún se encuentra lejos de ser cifras alentadoras para la construcción. Se contó con una mano de obra de 948.736 trabajadores para los niveles de riesgo II, III, IV, V, reportando ante las administradoras de riesgos laborales un total de 80.402 accidentes de trabajo en el año y 
ocupando el cuarto lugar en cuanto a los sectores productivos del país que mayor niveles de accidentalidad imponen a la fuerza trabajadora del país.

Es por ello que se requiere especial énfasis en la forma en la que nos encontramos desarrollando y llevando a cabo los sistemas de prevención para una actividad que nos está dejando en promedio 100 trabajadores fallecidos al año, la más alta en cuanto a mortalidad en nuestro país.

\subsection{Sistema de gestion de seguridad y salud en el trabajo}

El Sistema de Gestión de la Seguridad y Salud en el Trabajo SG-SST, tiene como propósito la estructuración de la acción conjunta entre el empleador y los trabajadores, en la aplicación de las medidas de Seguridad y Salud en el Trabajo (SST) a través del mejoramiento continuo de las condiciones y el medio ambiente laboral, y el control eficaz de los peligros y riesgos en el lugar de trabajo.

El Sistema de Gestión de la Seguridad y Salud en el Trabajo SG-SST consiste en el desarrollo de un proceso lógico y por etapas, basado en la mejora continua y que incluye la política, la organización, la planificación, la aplicación, la evaluación, la auditoría y las acciones de mejora con el objetivo de anticipar, reconocer, evaluar y controlar los riesgos que puedan afectar la seguridad y la salud en el trabajo [5].

Los Sistemas de gestión de Seguridad y Salud en el Trabajo en Colombia se encuentran reglamentados bajo las disposiciones emanadas por el ministerio del trabajo y su Decreto 1072 de 2015 -

Decreto único reglamentario del sector trabajo y la resolución 0312 de 2019, por la cual se definen los estándares mínimos del sistema de gestión de seguridad y salud en el trabajo, según el número de empleados y el nivel de riesgo al que se encuentren afiliados al sistema general de riesgos laborales.

\subsubsection{Ciclo de Deming}

Es una estrategia de mejora continua de la calidad en cuatro pasos, basada en un concepto idea- do por Walter A. Shewhart. Es muy utilizado por los sistemas de gestión de la calidad (SGC).

Herramienta de mejora continua, también conocido como el ciclo de Deming, ciclo de control o simplemente ciclo PHVA (Planear, Hacer, Verificar y Actuar).

Los resultados de la implementación de este ciclo permiten a las empresas una mejora integral de la competitividad, de los productos y servicios, mejorando continuamente la calidad, reduciendo los costos, optimizando la productividad, reduciendo los precios, incrementando la participación del mercado y aumentando la rentabilidad de la empresa u organización [6]. Fig. 1.

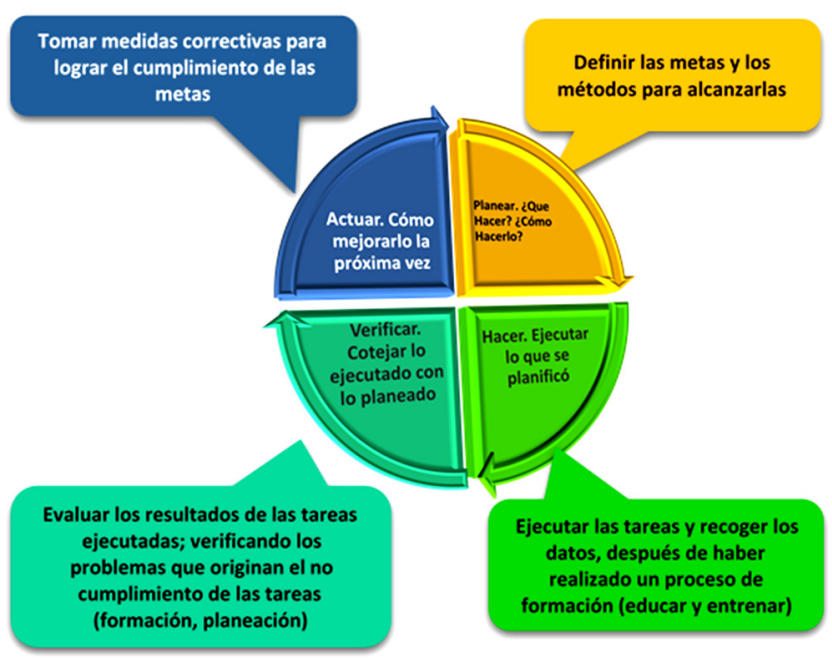

Fig. 1. Ciclo de mejora continua - PHVA.

\subsubsection{Normatividad colombiana en seguridad y salud en el trabajo (Tabla I).}

\subsubsection{Actividades a desarrollar en materia de se- guridad y salud en el trabajo, según la resolucion 0312 de 2019.}

Actualmente en Colombia y desde febrero de 2019, entro en vigencia la Resolución 0312 por la cual se definen los estándares mínimos de cumplimiento para el Sistema de Gestión de Seguridad y salud en el trabajo, dependiendo el número de trabajadores y el nivel de riesgo de la empresa según su actividad económica. 
Tabla I. Normatividad Colombiana para el sistema de gestión de seguridad y salud en el trabajo.

\begin{tabular}{|c|c|c|c|c|}
\hline Emisor & $\begin{array}{c}\text { Jerarquia de la } \\
\text { norma }\end{array}$ & Numero & Año & Titulo \\
\hline $\begin{array}{l}\text { Congreso de } \\
\text { Colombia }\end{array}$ & Ley & 1010 & 2006 & $\begin{array}{c}\text { Por medio de la cual se adoptan medidas para } \\
\text { prevenir, corregir y sancionar el acoso laboral y otros } \\
\text { hostigamientos en el marco de las relaciones de } \\
\text { trabajo }\end{array}$ \\
\hline $\begin{array}{l}\text { Congreso de } \\
\text { Colombia }\end{array}$ & Código & $\begin{array}{l}\text { Código } \\
\text { sustantivo } \\
\text { del trabajo }\end{array}$ & 1951 & Regulación de Relaciones Laborales \\
\hline $\begin{array}{l}\text { Ministerio de la } \\
\text { Protección } \\
\text { Social }\end{array}$ & Resolución & 1401 & 2007 & $\begin{array}{l}\text { Por la cual se reglamenta la investigación de } \\
\text { incidentes y accidentes de trabajo. }\end{array}$ \\
\hline $\begin{array}{l}\text { Ministerio de la } \\
\text { Protección } \\
\text { Social }\end{array}$ & Resolución & 2346 & 2007 & $\begin{array}{c}\text { Por la cual se regula la práctica de evaluaciones } \\
\text { médicas ocupacionales y el manejo y contenido de las } \\
\text { historias clínicas ocupacionales. }\end{array}$ \\
\hline $\begin{array}{l}\text { Ministerio de la } \\
\text { Protección } \\
\text { Social }\end{array}$ & Resolución & 2646 & 2008 & $\begin{array}{l}\text { Por la cual se establecen disposiciones y se definen } \\
\text { responsabilidades para la identificación, evaluación, } \\
\text { prevención, intervención y monitoreo permanente de } \\
\text { la exposición a factores de riesgo psicosocial en el } \\
\text { trabajo y para la determinación del origen. }\end{array}$ \\
\hline $\begin{array}{l}\text { Ministerio del } \\
\text { Trabajo }\end{array}$ & Resolución & 652 & 2012 & $\begin{array}{c}\text { Por la cualquier se establece la conformación y } \\
\text { funcionamiento del Comité de Convivencia Laboral } \\
\text { en entidades públicas y empresas privadas y se di tan } \\
\text { otros disposiciones. }\end{array}$ \\
\hline $\begin{array}{l}\text { Ministerio del } \\
\text { Trabajo }\end{array}$ & Resolución & 312 & 2019 & $\begin{array}{c}\text { Por la cual se definen los Estándares Mínimos del } \\
\text { Sistema de Gestión de la Seguridad y Salud en el } \\
\text { Trabajo SG-SST }\end{array}$ \\
\hline $\begin{array}{l}\text { Ministerio del } \\
\text { Trabajo }\end{array}$ & Decreto & 1072 & 2015 & Decreto Único Reglamentario del Sector Trabajo \\
\hline $\begin{array}{l}\text { Ministerio del } \\
\text { Trabajo }\end{array}$ & Resolución & 1409 & 2012 & $\begin{array}{l}\text { Por la cual se establece el reglamento de seguridad } \\
\text { para protección contra caídas en trabajo en alturas. }\end{array}$ \\
\hline $\begin{array}{l}\text { Ministerio del } \\
\text { Trabajo y } \\
\text { Seguridad } \\
\text { Social }\end{array}$ & Decreto - Ley & 1295 & 1994 & $\begin{array}{c}\text { Por el cual se determina la organización y } \\
\text { administración del Sistema General de Riesgos } \\
\text { Profesionales }\end{array}$ \\
\hline $\begin{array}{l}\text { Ministerio del } \\
\text { Trabajo y } \\
\text { Seguridad } \\
\text { Social }\end{array}$ & Resolución & 1792 & 1990 & $\begin{array}{c}\text { Por la cual se adoptan valores límites permisibles } \\
\text { para la exposición ocupacional al ruido. }\end{array}$ \\
\hline $\begin{array}{l}\text { Ministerio del } \\
\text { Trabajo y } \\
\text { Seguridad } \\
\text { Social }\end{array}$ & Resolución & 2013 & 1986 & $\begin{array}{c}\text { Por la cual se reglamenta la organización y } \\
\text { funcionamiento de los Comités de Medicina, Higiene } \\
\text { y Seguridad Industrial en los lugares de trabajo }\end{array}$ \\
\hline $\begin{array}{l}\text { Ministerio del } \\
\text { Trabajo y } \\
\text { Seguridad } \\
\text { Social }\end{array}$ & Resolución & 2400 & 1979 & $\begin{array}{c}\text { Por la cual se establecen algunas disposiciones sobre } \\
\text { vivienda, higiene y seguridad en los establecimientos } \\
\text { de trabajo. }\end{array}$ \\
\hline
\end{tabular}


Se clasificaron las empresas en 3 grupos de la siguiente manera:

- Estándares mínimos para empresas, empleadores y contratantes con diez (10) o menos trabajadores, clasificadas con riesgo I, II o III.

- Estándares mínimos para empresas de once (11) a cincuenta (50) trabajadores clasificadas con riesgo I, II o III.

- Estándares mínimos para empresas de más de cincuenta (50) trabajadores clasificadas con riesgo I, II, III, IV o V y de cincuenta (50) o menos trabajadores con riesgo IV o V.

ESTÁNDARES MÍNIMOS PARA EMPRESAS, EMPLEADORES Y CONTRATANTES CON DIEZ (10) O MENOS TRABAJADORES, CLASIFICADAS CON RIESGO I, II O III.

Las empresas que cumplan con estas características estarán en la obligación de llevar a cabo las siguientes 7 actividades junto con sus respectivas evidencias de cumplimiento:

1. Asignación de persona que diseña el Sistema de Gestión de SST

2. Afiliación al Sistema de Seguridad Social Integral

3. Capacitación en SST

4. Plan Anual de Trabajo

5. Evaluaciones médicas ocupacionales

6. Identificación de peligros; evaluación y valoración de riesgos

7. Medidas de prevención y control frente a peligros/riesgos identificados

Adicional, el diseño del Sistema de Gestión de SST para empresas de diez (10) o menos trabajadores clasificadas con riesgo I, II o III, podrá ser realizado por técnicos en Seguridad y Salud en el Trabajo (SST) o en alguna de sus áreas, con licencia vigente en Seguridad y Salud en el Trabajo, que acrediten mínimo un (1) año de experiencia certifi- cada por las empresas o entidades en las que laboraron en el desarrollo de actividades de Seguridad y Salud en el Trabajo y que acrediten la aprobación del curso de capacitación virtual de cincuenta (50) horas en SST [7].

\section{ESTÁNDARES MÍNIMOS PARA EMPRESAS DE ONCE (11) A CINCUENTA (50) TRABAJADORES CLASIFICADAS CON RIESGO I, II O III.}

Las empresas que cumplan con estas características estarán en la obligación de llevar a cabo las siguientes 21 actividades junto con sus respectivas evidencias de cumplimiento:

1. Asignación de persona que diseña el Sistema de Gestión de SST

2. Asignación de recursos para el Sistema de Gestión de SST

3. Afiliación al Sistema de Seguridad Social Integral

4. Conformación y funcionamiento del COPASST

5. Conformación y funcionamiento del Comité de Convivencia Laboral.

6. Programa de capacitación

7. Política de Seguridad y Salud en el Trabajo

8. Plan Anual de Trabajo

9. Archivo y retención documental del Sistema de Gestión de SST

10.Descripción socio demográfica y Diagnóstico de condiciones de salud

11. Actividades de medicina del trabajo y de prevención y promoción de la salud

12. Evaluaciones médicas ocupacionales

13. Restricciones y recomendaciones médicas laborales

14. Reporte de accidentes de trabajo y enfermedades laborales

15. Investigación de incidentes, accidentes de trabajo y enfermedades cuando sean diagnosticadas como laborales

16.Identificación de peligros y evaluación y valoración de riesgos 
17.Mantenimiento periódico de instalaciones, equipos, máquinas y herramientas

18.Entrega de los elementos de protección personal - EPP y capacitación en uso adecuado

19.Plan de prevención, preparación y respuesta ante emergencias

20.Brigada de prevención, preparación y respuesta ante emergencias

21. Revisión por la alta dirección.

El diseño e implementación del Sistema de Gestión de SST, para empresas de once (11) a cincuenta (50) trabajadores clasificadas en riesgo I, II o III, podrá ser realizado por tecnólogos en SST o en alguna de sus áreas, con licencia vigente en SST, que acrediten mínimo dos (2) años de experiencia certificada por las empresas o entidades en las que laboraron en el desarrollo de actividades de Seguridad y Salud en el Trabajo y el curso de capacitación virtual de cincuenta (50) horas en SST [7].

ESTÁNDARES MÍNIMOS PARA EMPRESAS DE MÁS DE CINCUENTA (50) TRABAJADORES CLASIFICADAS CON RIESGO I, II, III, IV O V Y DE CINCUENTA (50) O MENOS TRABAJADORES CON RIESGO IV O V.

Las empresas que cumplan con estas características estarán en la obligación de llevar a cabo las siguientes 60 actividades junto con sus respectivas evidencias de cumplimiento:

1. Asignación de una persona que diseñe e implemente el Sistema de Gestión de SST

2. Asignación de responsabilidades en SST

3. Asignación de recursos para el Sistema de Gestión en SST

4. Afiliación al Sistema de Seguridad Social Integral

5. Identificación de trabajadores que se dediquen en forma permanente a actividades de alto riesgo y cotización de pensión especial

6. Conformación y funcionamiento del COPASST

7. Capacitación de los integrantes del COPASST

8. Conformación y funcionamiento del Comité de Convivencia Laboral
9. Programa de capacitación anual

10.Inducción y reinducción en SST

11. Curso Virtual de capacitación de cincuenta (50) horas en SST

12.Política de Seguridad y Salud en el Trabajo

13.Objetivos de SST

14.Evaluación Inicial del Sistema de Gestión

15.Plan Anual de Trabajo

16. Archivo y retención documental del Sistema de Gestión de SST

17.Rendición de cuentas

18. Matriz legal

19. Mecanismos de comunicación

20.Identificación y evaluación para la adquisición de bienes y servicios

21. Evaluación y selección de proveedores y contratistas

22.Gestión del cambio

23. Descripción sociodemográfica y Diagnóstico de condiciones de salud de los trabajadores

24. Actividades de medicina del trabajo y de prevención y promoción de la Salud

25.Perfiles de cargos

26. Evaluaciones médicas ocupacionales

27. Custodia de las historias clínicas

28. Restricciones y recomendaciones médico laborales

29. Estilos de vida y entorno saludable

30.Servicios de higiene

31.Manejo de Residuos

32. Reporte de accidentes de trabajo y enfermedades laborales

33. Investigación de incidentes, accidentes de trabajo y las enfermedades cuando sean diagnosticadas como laborales

34.Registro y análisis estadístico de accidentes de trabajo y enfermedades laborales

35.Frecuencia de accidentalidad

Rev. Ingeniería, Matemáticas y Ciencias de la Información Vol. 8 / Núm. 15 / enero - junio de 2021; pág. 45-53 
36.Severidad de accidentalidad

37.Proporción de accidentes de trabajo mortales

38.Prevalencia de la enfermedad laboral.

39.Incidencia de la enfermedad laboral

40.Ausentismo por causa médica

41.Metodología para identificación de peligros, evaluación y valoración de riesgos

42. Identificación de peligros y evaluación y valoración de riesgos con participación de todos los niveles de la empresa

43.Identificación de sustancias catalogadas como carcinógenas o con toxicidad aguda.

44. Mediciones ambientales

45. Medidas de prevención y control frente a peligros/riesgos identificados

46. Aplicación de medidas de prevención y control por parte de los trabajadores

47.Procedimientos e instructivos internos de seguridad y salud en el trabajo

48. Inspecciones a instalaciones, maquinaria o equipos

49. Mantenimiento periódico de las instalaciones, equipos, máquinas y herramientas

50.Entrega de los Elementos de Protección Personal (EPP) y capacitación en uso adecuado

51.Plan de prevención, preparación y respuesta ante emergencias

52. Brigada de prevención, preparación y respuesta ante emergencias

53. Definición de indicadores del Sistema de Gestión de Seguridad y Salud en el Trabajo

54.Auditoría anual

55.Revisión por la alta dirección. Alcance de la auditoría del Sistema de Gestión

56.Planificación de la auditoría con el COPASST

57. Acciones preventivas y/o correctivas

58. Acciones de mejora conforme a revisión de la Alta Dirección
59. Acciones de mejora con base en investigaciones de accidentes de trabajo y enfermedades laborales

\section{Plan de mejoramiento}

El diseño e implementación del Sistema de Gestión de SST, para empresas de más de cincuenta (50) trabajadores, clasificadas con riesgo I, II, III, IV o V y las de (50) o menos trabajadores con riesgo IV o V, podrá ser realizado por profesionales en SST, profesionales con posgrado en SST; que cuenten con licencia en SST vigente y el curso de capacitación virtual de cincuenta (50) horas en SST, quienes igualmente están facultados para asesorar, capacitar, ejecutar o diseñar el Sistema de Gestión de SST en cualquier empresa o entidad, sin importar la clase de riesgo, número de trabajadores o actividad económica [7].

Ya que la construcción es una actividad que supone la exposición a una gran cantidad de riesgos laborales, algunos de ellos graves y la singularidad que representa, con relación al resto de los sectores, se debe principalmente a la movilidad, temporalidad y provisionalidad de muchos de los procesos, que hacen que esta actividad tenga una consideración diferente en el ámbito de la prevención de riesgos laborales, es por esto que su nivel de riesgo en todos los casos será el $\mathrm{V}$, viéndose obligados los contratistas y empresas constructoras de nuestro país a cumplir a cabalidad con los 60 ítems de verificados emanados por el ministerio del trabajo según la resolución 0312 de 2019.

\subsubsection{Riesgos laborales en la construccion}

A. Caídas:

Las caídas pueden ser de varios tipos. Las caídas a distinto nivel, ocurren especialmente en la realización de trabajos en tejados y cubiertas, huecos exteriores o interiores, y andamios.

También son frecuentes las caídas al mismo nivel, provocadas por tropezones, pisar en terreno inestable o resbalones.

B. Caída de objetos o derrumbes:

Este tipo de accidentes pueden deberse a la caída de elementos debido a la inestabilidad de la 
estructura. Aquí entrarían las caídas de edificios, muros, materiales de construcción, etc. Por ello siempre es necesario una buena colocación de estos materiales y, en caso de observar cualquier indicio de inestabilidad, comunicarlo al responsable de la obra.

\section{Orden y limpieza:}

Muchos accidentes se producen por golpes y caídas que podrían haberse evitado con un ambiente ordenado y recogido. A menudo, un suelo resbaladizo o materiales fuera de sitio son los que provocan estos daños.

\section{Proyección de partículas:}

Este tipo de lesiones pueden producirse por pequeños fragmentos o partículas de un material que han sido proyectadas por una máquina o herramienta.

\section{E. Riesgo eléctrico:}

La construcción, por su carácter de provisionalidad, hace que tenga un riesgo especial por lo que respecta a la instalación eléctrica. Estas instalaciones suelen ser provisionales, están a menudo al aire libre, se componen de material reutilizable, entre otros aspectos. Todo esto hace que haya mayores probabilidades de accidente que en otros sectores.

\section{F. Vuelco de maquinaria:}

En la construcción es necesario el uso de maquinaria pesada. En ocasiones el terreno sobre el que se está realizando una obra puede ser inestable, lo que puede ocasionar el vuelco de la máquina, atrapando al operario o alguna parte de su cuerpo [8].

Según como lo indica el Ministerio del trabajo en su artículo "Que no le pase: Centenares de empresas han sido multadas por no pagar salud a empleados." Para el año 2018 el ministerio sancionó en 370 oportunidades y estas multas ascendieron a $13 \mathrm{mil}$ millones de pesos. Para el mes de septiembre de 2019 se contabilizaban 289 quejas con sanciones por 9 mil millones de pesos, dichas multas por evadir los respectivos aportes a seguridad social de sus trabajadores y no cumplir con las obligaciones en materia de seguridad y salud en el trabajo.

\section{CONCLUSiOnes}

De acuerdo a los resultados obtenidos en el presente artículo, se concluye que el sector de la construcción es uno de los sectores que mayor atención requieren en materia de seguridad y salud en el trabajo en Colombia por parte de las autoridades, sector que debido a sus altos índices de accidentalidad requiere una metodología aplicable para los sistemas de gestión más rigurosa y exhaustiva.

Por otra parte, la ocurrencia de los accidentes de trabajo han sido generados en mayor parte por faltas de control, seguido de actos inseguros; que como lo define Sibaja, se deben a todo tipo de acciones $\mathrm{u}$ omisiones cometidas por las personas que posibilitan que se produzcan los accidentes [9].

En la actualidad se ha dado un paso evolutivo en cuanto a la normatividad en materia de riesgos laborales con la que se ha podido establecer la necesidad de implementar un Sistema de Gestión de Seguridad y Salud en el trabajo, en el cual los profesionales en la materia tienen el reto de garantizar condiciones dignas y disminuir las barreras para la participación y la igualdad.

Se hace indispensable el trabajo mancomunado entre los empleadores, que son los responsables por llevar a cabo las actividades que garanticen a sus trabajadores unas condiciones laborales óptimas para desempeñar sus funciones, reduciendo al máximo la exposición a posibles accidentes de trabajo, las administradoras de riesgos laborales, que son quienes apoyan a los empleadores en este proceso y de alguna manera $\mathrm{u}$ otra no garantizan el apoyo y seguimiento adecuado a las empresas afiliadas y por último, el papel importante del ministerio del trabajo en cuanto al seguimiento y retroalimentación a las actividades llevadas a cabo por los empleadores en sus empresas.

\section{RECOMENDACIONES}

Por los resultados obtenidos en el presente artículo, se sugiere para el sector de la construcción en Colombia efectuar un sistema de gestión de seguridad y salud en el trabajo, cuya finalidad se base en la adecuada identificación, evaluación y control de los diferentes peligros y riesgos que se 
hayan presentes en los lugares de trabajo, encaminado a la protección de las partes del cuerpo más afectadas durante la ejecución de las actividades; así como también proporcionar a los trabajadores la formación e información necesaria para el desempeño de las funciones en óptimas condiciones de seguridad, en donde la inducción diaria sobre el desarrollo de las actividades a través de una seguridad basada en el comportamiento sea primordial para todos los trabajadores sin excepción alguna, y además emprender programas de sensibilización y concientización de tomar la seguridad como un valor fundamental.

Asimismo, se recomienda a los empleadores, elaborar y estandarizar el análisis y los procedimientos de trabajo, especialmente las tareas críticas, las cuales se deben identificar mediante un adecuado estudio de riesgos, que permita valorarlos para diseñar programas de gestión que faciliten su intervención y control.

Se considera que la planeación es una de las etapas fundamentales que toda organización debe realizar para la prevención de los accidentes laborales, se trata de reunir en un documento, de forma clara y detallada, toda la información indispensable para realizar una actividad de manera preventiva y sistemática, coherente y eficaz, frente a los riesgos presentes en la actividad de una empresa. El tiempo y el dinero manejados en esta actividad se recuperan obteniendo resultados efectivos y rápidos; es necesario tener claro que no basta con que se plasme en un papel, sino que se lleve también a la práctica y para esto es fundamental que se supervise el desarrollo adecuado del mismo. Los datos reflejados en el presente artículo demuestran la necesidad de emprender el tema de la seguridad y la salud en el trabajo en todas sus dimensiones y con la participación de todos los integrantes de la organización, (empleadores, administradoras de riesgos laborales, contratistas, trabajadores e instituciones de formación) y de esta forma se pueda realizar una propuesta común, que tenga como objetivo mejorar la calidad de vida de los trabajadores del sector, disminuyendo los incidentes de trabajo.

\section{REFERENCIAS}

[1] Fasecolda. Obtenido de https://sistemas. fasecolda.com/rldatos/Reportes/xClaseGrupo Actividad.aspx. 2020.

[2] P. M. Grisales, Repositorio uniandes. Obtenido de https:/ / repositorio.uniandes.edu.co/bitstream/ handle/1992/11339/u442497.pdf? sequence=1. 2011.

[3] Salud, M.d. Minsalud.gov.co. Obtenido de https:/ / www.minsalud.gov.co/sites/rid/Lists/ BibliotecaDigital/RIDE/DE/DIJ/Ley-1562-de2012.pdf. 2012.

[4] O. C. Marquez, eben-spain.org. Obtenido de http:/ / www.eben-spain.org/docs / Papeles / X/ Armnguo-Olivr.pdf. 2020.

[5] Mincultura. Ministerio de Cultura. Obtenido de https://www.mincultura.gov.co/prensa/noticias / Documents / Gestion-humana / PLAN\%20SG-SST\%202017.pdf. 2017.

[6] H. Rodríguez, wordpress.com. Obtenido de https:/ /ntc6001.wordpress.com/2015/07/31/ciclophva/ 2015.

[7] Trabajo, M. d. Resolucion 0312. Obtenido de Resolucion 0312: https://id.presidencia.gov.co/ Documents/190219_Resolucion0312Estandares MinimosSeguridadSalud.pdf. 2019.

[8] Prevencionar. Prevencionar.com.co. Obtenido de Prevencionar.com.co:

https:/ / prevencionar.com.co/2017/11/17/riesgos-laborales-la-construccion/. 2017.

[9] R. C. Sibaja, books.google.com.co. Obtenido de books.google.com.co:

https://books.google.com.co/books?id $=Y 35 \mathrm{TDM} 74 \mathrm{KmUC} \&$ printsec $=$ frontcover $\& \mathrm{hl}=\mathrm{e}$ s\&source $=$ gbs_ge_summary_r\&cad $=0 \# \mathrm{v}=$ onepage $\&$ $\mathrm{q} \& \mathrm{f}=$ false. 2002. 
